\title{
Stochastic models of the growth dynamics of some dendritic cells
}

\author{
Khairia El-Said El-Nadi \\ Khairia_el_said@hotmail.com \\ Faculty of Science Alexandria University, Alexandria, Egypt.
}

\begin{abstract}
Different models of tumor growth are considered. Some mathematical methods are developed to analyze the dynamics of mutations enabling cells in cancer patients to metastize. The mathematical models consist of some stochastic dynamical systems describing tumor cells and immune effectors. It is also considered a method to find the ideal outcome of some treatments. Some different types of dendritic cells are considered. The obtained results will help to find some suitable treatments, which can be successful in returning an aggressive tumor to its passive,non-immune evading state. The principle goal of this paper is to find ways to treat the cancer tumors before they can reach an advanced stage developmen.
\end{abstract}

Keywords: Stochastic dynamical systems, tumor cells, treatment of cancer.

AMS Subject Classifications: 92B05, 37C45. 


\section{Introduction}

In this paper some deterministic and stochastic mathematical models are considered, which explain the interaction of the immune system and cancer.

Any treatment, which can improve the bodies own immune response is called the immunotherapy.

The treatment by cytotoxic chemotherapy helps to kill rapidly dividing cells, but it can harm normal tissues at the same time. The use of immunotherapy in conjuction with cytotoxic chemotherapy is known as bio chemotherapy. The bio chemotherapy treatment is obtained by using the immunological drug Interleukin-2. The Interleukin-2 at time $\mathrm{t}$ is denoted by I(t). The cells, which can not kill other cells are called naive T-cells. The number of naive t-cells at time $\mathrm{t}$ is denoted by $T_{N}(t)$. The naive t-cells will have the ability to kill other cells, if activated by antigen presenting cells. The most important type of antigen presenting cells are known as immature dendritic cells, immunogenic dendritic cells and to tolerogenic dendritic cells. All cells population are assumed to be antigen specific.

In section 2 , we shall write the most recent mathematical models, which incorporate some different cells and the cytosine IL-2.

In section 3 , we generalize the models in section 2 to stochastic dynamical systems.

\section{2. deterministic models and cancer}

There are various mathematical models of cancer and immune response [1-6]. As an example, let $\phi(t)$ be the population of the immunogenic dendritic cells at time $\mathrm{t}$ and $\psi(t)$ be the Immature dendritic cells. It is assumed that the cells $\phi(t)$ are produced at a constant rate a by the Immature dendritic cells $\psi(t)$. It also decays at a constant rate $\omega$. This yields the following equation:

$$
\frac{d \phi}{d t}=a \psi(t)-\omega \phi(t)
$$


Let $M(t)$ be the chemotherapy drug concentration in the blood stream at time t. The functions $M(t)$ and $I(t)$ decay exponentially with respect to $t$;

$$
M(t)=M(0) e^{-\omega_{1} t}, I(t)=I(0) e^{-\omega_{2} t},
$$

where $\omega_{1}$ and $\omega_{2}$ are positive constants.

The tumor cell population $T(t)$ grows logistically in the absence of immune response, $[7,8]$. The function $T(t)$ satisfies the equation

$$
\frac{d T(t)}{d t}=a_{1} T\left(1-b_{1} T\right)-D(t) T
$$

where

$$
D(t)=b \frac{[L(t) / T(t)]^{\alpha}}{s+[L(t) / T(t)]^{\alpha}},
$$

and $L(t)$ is the number of cells, which combat a specific invader at time $t$.

Let $\eta(t)$ be the number of tolerogenic dendritic cells. following $[9,10,11]$, the function $\eta$ has linear natural death term and satisfy the equation

$$
\frac{d \eta}{d t}=F(t) \psi(t)-a_{2} \eta(t)
$$

where $\mathrm{F}$ is given by

$$
F(t)=b_{2} \frac{T_{R}(t)}{c_{1}+T_{R}(t)}+b_{3} \frac{I(t)}{c_{2}+I(t)}
$$

The function $T_{R}$ is the number of the so-called regularity T-cells at time t. The relation between $T_{R}(t)$ and $T_{N}(t)$ is given by:

$$
\begin{aligned}
\frac{d T_{R}(t)}{d t} & =b_{4} \eta(t) T_{N}(t) \\
& +b_{5} T_{R}(t) \frac{I(t)}{c_{3}+I(t)}-a_{3} T_{R}(t) .
\end{aligned}
$$

The function $T_{N}(t)$ satisfy the following equation;

$$
\frac{d T_{N}(t)}{d t}=a_{4}-\frac{\omega_{3}}{c_{4}+I_{N}(t)} T_{N}(t)
$$

All the parameters $a, b, a_{1}, \ldots a_{4}, b_{1}, \ldots, b_{5}, c_{1}, \ldots, c_{4}$ are nonnegative constants. 


\section{Stochastic models}

It is preferred to find ways to treat tumors before they can reach an advanced stage of development.

Let us study the immunogenic dendritic cells $\phi(t)$.

To be more realistic, we consider the effect of the concentration of the chemotherapy $\mathrm{M}(\mathrm{t})$, and assume that $\{\phi(t)\}$ is a stochastic process satisfying a stochastic differential equation

$$
\begin{aligned}
d \phi(t) & =[a \psi(t)-\omega \phi(t)] d t \\
& -k \phi(t)\left[1-e^{-M(t)}\right] d t+ \\
& +\sigma d W(t)
\end{aligned}
$$

where $\sigma$ is the volatility of the process $\{\phi(t)\}$ and $\mathrm{W}(\mathrm{t})$ is a Wiener process with zero mean and unit variance.

The function $\psi(t)$ satisfies the equation

$$
\frac{d \psi(t)}{d t}=g(T(t))-F(t) \psi(t)-b \psi(t)
$$

where $g$ increases monotonically with respect to $\mathrm{T}(\mathrm{t}),[8,9]$.

equation (3.1) can be written in the form

$$
d X(t)=g_{1}(t) d t+g_{2}(t) d W(t)
$$

where

$$
\begin{gathered}
g_{1}(t)=a \psi(t) g_{3}(t)-\phi(0) \frac{d g_{3}(t)}{d t}, \\
g_{2}(t)=\sigma g_{3}(t), \\
g_{3}(t)=\exp \left[(w+k) t-k \int_{0}^{t} e^{-M(s) d s}\right], \\
X(t)=g_{3}(t)[\phi(t)-\phi(0)],
\end{gathered}
$$

$\mathrm{a}, \mathrm{w}, \mathrm{k}$ and $\alpha$ are positive constants.

It is supposed that $\phi(0)$ is deterministic. It is clear that

$$
x(t)=\int_{0}^{t} g_{1}(s) d s+\int_{0}^{t} g_{2}(s) d W(s)
$$




$$
\phi(t)=g_{3}^{-1}(t) X(t)+\phi(0) .
$$

According to Feynman-Kac formula [9], we get

$$
\begin{gathered}
\frac{\partial u(x, t)}{\partial t}=\frac{1}{2} g_{2}^{2}(t) \frac{\partial^{2} u(x, t)}{\partial x^{2}}+g_{1}(t) \frac{\partial u(x, t)}{\partial x}, \\
u(x, 0)=f(x),
\end{gathered}
$$

where

$$
u(x, t)=E[f(x+X(t)],
$$

$(\mathrm{E}(\mathrm{Y})$ is the expectation of $\mathrm{Y})$.

If $\mathrm{f}$ is continuous and bounded on $(-\infty, \infty)$, then the solution of $(3.4),(3.5)$ is given by;

$$
u(x, t)=\frac{1}{\sqrt{4 \pi g(t)}} \int_{-\infty}^{\infty} e^{\frac{-(y-\xi)^{2}}{4 g(t)}} f(\xi) d \xi
$$

where

$$
\begin{aligned}
& g(t)=\frac{1}{2} \int_{0}^{t} g_{2}^{2}(s) d s, \\
& y=x+\int_{0}^{t} g_{1}(s) d s .
\end{aligned}
$$

using (3.6), one gets that the characteristic function of the stochastic process $\{X(t)\}$ is given by

$$
E\left[e^{i \lambda X(t)}\right]=\exp \left[i \lambda \int_{0}^{t} g_{1}(s) d s-\lambda^{2} g(t)\right] .
$$

From (3.3), we get

$$
\begin{gathered}
E[\phi(t)]=g_{3}^{-1}(t) h(t), \\
h(t)=a \int_{0}^{t} g_{3}(s) \psi(s) d s+\phi(0) .
\end{gathered}
$$

From (3.7), one gets

$$
E\left[e^{i \lambda g_{3}(t) \phi(t)}\right]=\exp \left[\frac{-h^{2}(t)}{4 g(t)}-g(t)\left\{\lambda-\frac{i h(t)}{2 g(t)}\right\}\right] .
$$

Using the last formula, we find that all the moments of the stochastic process $\{\phi(t)\}$ are given by :

$$
\left(i g_{3}(t)\right)^{n} E\left[\phi^{n}(t)\right]=(-1)^{n} g^{\frac{n}{2}}(t) H_{n}\left(\frac{-i h(t)}{2 \sqrt{g(t)}}\right)
$$


where $H_{n}(x)$ is the Hermite polynomial.

The variance of $\phi$ is given by $\operatorname{Var}[\phi(t)]=2 g_{3}^{-2}(t) g(t)$.

Our stochastic model,[10-16] should be viewed as an attempt to understand the growth dynamics of some dendritic cells and the effect of the chemotherapy.

In immunotherapy without IL-2 delivery, tumor-specific lymphocytes are reintroduced to the body after being inoculated with high concentrations of the cytokine IL-2. We add such treatment via immunotherapy to this model by shifting the initial lymphocyte population. (see $[17-23])$.

\section{References}

[1] Michael B. Atkins. Cytokine-bassed therapy and bio chemotherapy for advanced melanoma. Clinical Cancer research, 12:2353-2358, 2006.

[2] Jorge Carneiro, Tiago Paixzo, Dejan Milutinovic, Joao Sousa, Kalet Leona, Rui Gardner, and Jose Faro. Immunological Self-tolerance: Lessons from mathematical modeling. Journal of Computational and Applied Mathematics, 184: 77-100, 2004.

[3] Renee Fister and Jennifer Hughes Donnelly. Immunotherapy: An optimal control theory approach. Mathematical Biosciences and Engineering, 2: 499-510, 2005.

[4] Avner r Friedman. A hierarchy of cancer models and their mathematical challenges. Discrete and Continuous Dynamical Systems-Series B, 4: 147-159, 2004.

[5] Shea N. Gardner. A mechanistic, predictive model of dose-response curves for cell cycle phase-specific and nonspecific drugs. Cancer Research, 60: 1417-1425, 2000.

[6] Angela M. Thornton and Ethan M. Shevach. Suppressor effector function of CD4+CD25+immunoregulatory T-cells is antigen nonspecific. Journal of Immunology, 164: 183-190, 2000. 
[7] P.W.A. Dayananda, J.M.G. Taylor, D.G. Whiting, Prostate cancer: progression of prostate-specific antigen after external beam irradiation, Math. Biosci. 182 (2003) 127.

[8] J.M.G. Taylor, K. Griffith, H.M. Sandler, Definitions of biochemical failure in prostate cancer following radiation therapy, Int. J. Radiat. Oncol. Biol. Phys. 50 (2001) 1212.

[9] J. Michael Steele, Stochastic calculus and financial applications, Springer, New York, 270-273, 2000.

[10] Khairia El-Said El-Nadi, Asymptotic formulas for some cylindrical functions, Uspekhi Math. Nauk, Vol. 24, No.3, 143-147, 1969.

[11] Khairia El-Said El-Nadi, Asymptotic methods in difference differential equations and its applications. A bad. J. Numerical. Analysis, Vol. 9, No. 4, 873-883, 1970.

[12] Khairia El-Said El-Nadi, On some boundary-value problems in queuing theory, System and Control letters, Vol 2, No.5, 307-312, 1983.

[13] Khairia El-Said El-Nadi, On some stochastic parabolic differential equations in a Hilbert space, J. of Applied Mathematics and stochastic analysis, Vol. 2, 167-179, 2005.

[14] Khairia El-Said El-Nadi, On some stochastic differential equations and fractional Brownian motion, Int. J. of pure and applied Math., Vol. 24, No. 3, 415-425, 2005.

[15] Khairia El-Said El-Nadi, Asymptotic methods and some difference fractional differential equations, Int. J. Contemp. Math. Sci., Vol 1, No.1, 3-13, 2006.

[16] Khairia El-Said El-Nadi; On the stability of some stochastic integro partial differential equation, Int. J. Contemp. Math. Sciences, Val. 2, 2007, 1317-1326. 
[17] Mahmoud M. El-Borai, Khairia El-Said El-Nadi and Hoda A. Foad, On some fractional stochastic delay differential equations, Computers and Mathematics with Applications, $59,2010,1156-1170$.

[18] Khairia El-Said El-Nadi and Mahmoud M. El-Borai, Integrated Semi groups and Cauchy problem for some fractional abstract differential equations, Life Science Journal, 2013, 10(3), 793-798.

[19] Khairia El-Said El-Nadi and Mahmoud M. El-Borai, An inverse fractional abstract Cauchy problem with nonlocal conditions, Life Science Journal, 2013, 10(3) 1705-1709.

[20] Mahmoud M. El-Borai and Khairia El-Said El- Nadi, A stochastic model of the growth and diffusion of brain tumor cancer, Jokull Journal, vol. 64, Issue. 4, 2014, pp. 222-233.

[21] Mahmoud M. El-Borai and Khairia El-Said El-Nadi, A parabolic transform and some stochastic ill-posed problems, British Journal of Mathematics and Computer Science, $2015,9(5), 418-426$.

[22] Khairia El-Said El-Nadi, L.M. Fatehy, Nourhan Hamdy Ahmed, Marchall Olkin exponential with application on cancer stem cells, American Journal of Theoritical and Applied Statistics, 2017, 6(5-1), 1-7.

[23] Khairia El-Said El-Nadi, Mahmoud M. El-Borai, On the stability of some fractional dynamical models related to tumor cancer evaluation, Elixir International Journal, Elixir Adv. Math. 112(2017) 4889448899 